\title{
Polarization-dependent confocal Raman microscopy of an individual ZnO nanorod
}

\author{
Chih-Tao Chien, ${ }^{1}$ Ming-Chung Wu, ${ }^{1}$ Chun-Wei Chen,,${ }^{1, a)}$ Hung-Hsien Yang, ${ }^{2}$ Jih-Jen Wu, ${ }^{2}$ \\ Wei-Fang Su, ${ }^{1}$ Chauo-Sung Lin, ${ }^{1}$ and Yang-Fang $\mathrm{Chen}^{3}$ \\ ${ }^{1}$ Department of Materials Science and Engineering, National Taiwan University, Taipei 106, Taiwan \\ ${ }^{2}$ Department of Chemical Engineering, National Cheng Kung University, Tainan 70101, Taiwan \\ ${ }^{3}$ Department of Physics, National Taiwan University, Taipei 106, Taiwan
}

(Received 13 March 2008; accepted 10 May 2008; published online 2 June 2008)

\begin{abstract}
In this study, polarized-Raman scattering measurements of an individual $\mathrm{ZnO}$ nanorod were carried out by using a confocal microscope together with a high-resolution piezoelectric stage. A predominant $A_{1}$ (TO) mode at $378 \mathrm{~cm}^{-1}$ in the parallel polarization $\left(E_{\|}\right)$configuration and a predominant $E_{2}$ (high) mode at $438 \mathrm{~cm}^{-1}$ in the perpendicular polarization $\left(E_{\perp}\right)$ configuration demonstrate the strong polarization dependent Raman scattering signals of an individual $\mathrm{ZnO}$ nanorod. The Raman intensity images of the individual $\mathrm{ZnO}$ nanorods with different orientation configurations can also be obtained, which reflect the interplay between the local phonon behavior and geometric anisotropy. (c) 2008 American Institute of Physics. [DOI: 10.1063/1.2938701]
\end{abstract}

$\mathrm{ZnO}$ has a direct band gap of $3.37 \mathrm{eV}$ with a large exciton binding energy of $60 \mathrm{meV}$ at room temperature. Due to the strong binding energy of excitons, $\mathrm{ZnO}$-based semiconductors are recognized as very promising materials for micro- or nanooptoelectronic applications in the UV region. $^{1-5}$ One-dimensional nanometer-sized semiconductor materials, i.e., nanowires and nanorods, have recently attracted considerable attention due to consisting of a high surface-to-volume ratio, which can critically affect the electronic and optical properties. ${ }^{6-8}$ Large optical anisotropy in the well-aligned $\mathrm{ZnO}$ nanowires have been demonstrated, due to its high aspect ratio nature of the one-dimensional nanomaterial. ${ }^{9}$ Raman scattering is a very useful nondestructive measurement to probe the phonon behavior of materials, providing the information of crystal structure, lattice dynamics, and defects. Recently, investigations of Raman scattering on an individual GaN nanowire, ${ }^{10,11}$ nanorod, ${ }^{12}$ carbon nanotube, ${ }^{13} \mathrm{SiC}$ nanowire, ${ }^{14}$ and $\mathrm{CdSe}$ nanowire ${ }^{15}$ have been demonstrated. In this article, polarization-dependent Raman scattering measurements on individual $\mathrm{ZnO}$ nanorods were investigated by confocal Raman microscopy together with a high-resolution piezoelectric stage. Mapping of the Raman shift signal was also conducted to probe the local information of phonon behavior of an individual $\mathrm{ZnO}$ nanorod, correlating with its geometric and orientation-dependent anisotropy.

$\mathrm{ZnO}$ nanorods were grown on seeded indium tin oxide (ITO) substrates using chemical bath deposition. Formation of the seed layer on ITO was conducted by the dip-coating method using an aqueous solution of zinc acetate dihydrate $\left(\mathrm{Zn}\left(\mathrm{CH}_{3} \mathrm{COO}\right)_{2} \cdot 2 \mathrm{H}_{2} \mathrm{O}, \mathrm{ZnAc} \cdot 2 \mathrm{H}_{2} \mathrm{O}\right)$ and hexamethylenetetramine $\left(\mathrm{C}_{6} \mathrm{H}_{12} \mathrm{~N}_{4}, \mathrm{HMTA}\right)$. The scanning electron microscope (SEM) images of the as-grown $\mathrm{ZnO}$ nanorod array and an individual $\mathrm{ZnO}$ nanorod are shown in Figs. 1(a) and 1(b), respectively. The $\mathrm{ZnO}$ nanorods consist of a length of about $10 \mu \mathrm{m}$ and diameters ranging from 180 to $300 \mathrm{~nm}$, which were obtained by multiple bath growth in a fresh aqueous

${ }^{\text {a) }}$ Author to whom correspondence should be addressed. Electronic mail: chunwei@ntu.edu.tw. solution of $\mathrm{ZnAc} \cdot 2 \mathrm{H}_{2} \mathrm{O}$ and HMTA at $95^{\circ} \mathrm{C}$ for every $3 \mathrm{~h}$. The high-resolution transmission electron microscope (TEM) image of an individual $\mathrm{ZnO}$ nanorod and the corresponding selection area electron diffraction pattern are also shown in the Fig. 1(c). They reveal that the nanorod possesses the single-crystal structure and the lattice spacing of around $0.52 \mathrm{~nm}$ along the longitudinal axis direction ( $c$ axis) corresponds to the $d$ spacing of $\mathrm{ZnO}(001)$ crystal planes.

Raman scattering measurements were conducted with a confocal microscopy (WITec, CMR200, Germany) in the backscattering mode. The scanning confocal Raman images were collected using a high-resolution piezoelectric stage (PI). A He-Ne laser $\left(\lambda_{\text {exe }}=632.8 \mathrm{~nm}\right)$ is used as the excitation source. The polarization of the light was selected using a half-wave plate. The Raman image mapping was obtained by intensity integration of the spectra by recording the data with $200 \mathrm{~nm} / \mathrm{step}$ and an integration time of $1 \mathrm{~s} / \mathrm{step}$. In all cases, the laser beam was focused down with a $100 \times$ numerical aperture $=0.95$ objective (Olympus) and the focused laser beam was about $1 \mu \mathrm{m}$ in diameter, corresponding to about 4-5 pixels in the integrated Raman image.

According to the group theory, four Raman active modes of $A_{1}, E_{1}$, and $2 E_{2}\left(E_{2}^{\text {low }}\right.$ and $\left.E_{2}^{\text {high }}\right)$ are expected for the wurtzite-type $\mathrm{ZnO}$ structure, which belongs to the space group of $C_{6 v}^{4}{ }^{16}$ In addition, the polar nature of $A_{1}$ and $E_{1}$ modes leads to the splitting of phonon signals into transverse-optical $\left(A_{1 T}\right.$ and $\left.E_{1 T}\right)$ and longitudinal optical $\left(A_{1 L}\right.$ and $\left.E_{1 L}\right)$ components. For the individual $\mathrm{ZnO}$ nanorod, three different polarization configurations of $\bar{X}(Y, Y) X, \bar{X}(Z, Z) X$, and $\bar{X}(Y, Z) X$ according to the Porto notation were investigated, where the $c$ axis of the nanorod is set as the $Z$ axis. The term of $\bar{X}(Y, Z) X$ represents that the laser light is incident from the opposite $X$ direction with the $Y$ polarization, while the scattered signal is collected with the $Z$ polarization in the $X$ direction. The coordination of the single $\mathrm{ZnO}$ nanorod with respect to the incident/scattered light is shown in Fig. 2(a). Figure 2(b) shows the Raman shift spectra of an individual $\mathrm{ZnO}$ nanorod with different polarization configurations. For comparison, the Raman signal of the as-grown $\mathrm{ZnO}$ nanorod array (denoted as ensemble) was also mea- 
(b)

(a)
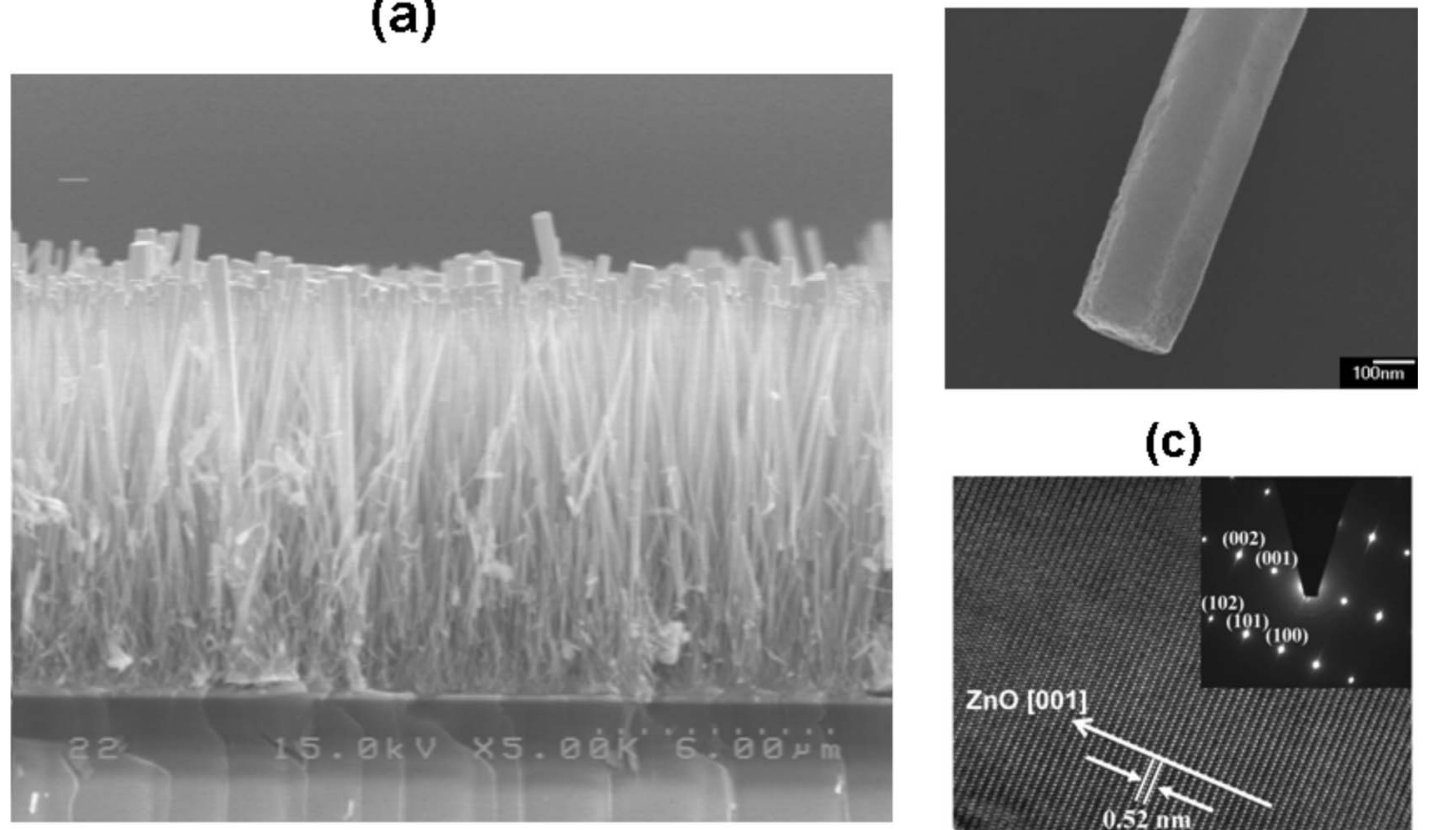

(c)

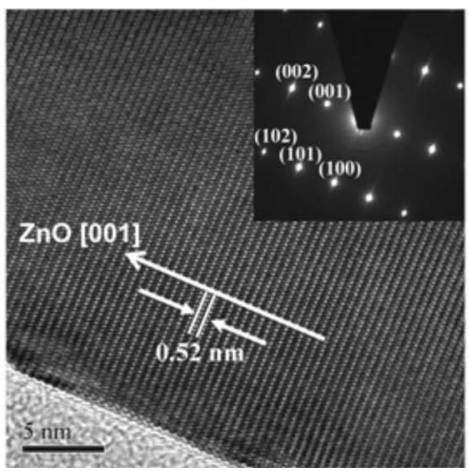

FIG. 1. (a) The SEM micrograph of the well-aligned $\mathrm{ZnO}$ nanorods. The scale bar is $6 \mu \mathrm{m}$. (b) The SEM image of an individual $\mathrm{ZnO}$ nanorod. The scale bar is $100 \mathrm{~nm}$. (c) High-resolution TEM image of an individual $\mathrm{ZnO}$ nanorod (growth along the $c$ axis) and the corresponding electron diffraction pattern.

sured. For the as-grown sample, the peaks at 331, 378, 410, and $438 \mathrm{~cm}^{-1}$ can be resolved, which can be assigned to $A_{1}$ (acoustic overtone), $A_{1}$ (TO), $E_{1}$ (TO), and $E_{2}$ (high) symmetry modes, respectively, which are consistent with those that reported the Raman shift signals of the bulk $\mathrm{ZnO}$ thin films or ensemble of nanostructures. ${ }^{17-19}$ For the Raman shift signals of the individual $\mathrm{ZnO}$ nanorod, strong polarization dependence on Raman signatures can be found. In the $\bar{X}(Y, Y) X$ configuration, where the incident/scattered photons

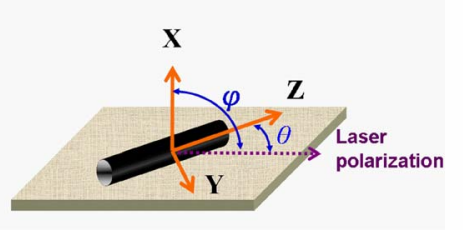

(a)

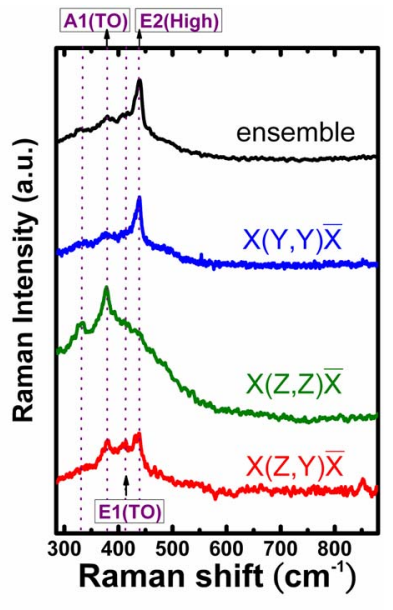

(b)
FIG. 2. (Color online) (a) The coordination of the single $\mathrm{ZnO}$ nanorod with respect to the incident/scattered light. $\theta(\varphi)$ is the angle between the laser polarization and the $Z$ axis ( $X$ axis). (b) The Raman shift spectra of the single $\mathrm{ZnO}$ nanorod with different polarization configurations of $\bar{X}(Y, Y) X$, $\bar{X}(Z, Z) X$, and $\bar{X}(Y, Z) X$ according to the Porto notation. The spectra of the ensemble represents the Raman scattering spectra of the as-grown $\mathrm{ZnO}$ nanorod array. orientation of the nanorod with respect to the polarization of
norod array.
Downloaded 04 Jun 2008 to 140.112.32.21. Redistribution subject to AIP license or copyright; see http://apl.aip.org/apl/copyright.jsp are polarized perpendicular to the $c$ axis of the nanorod (denoted as $E_{\perp}$ ), the peak at $438 \mathrm{~cm}^{-1}$ of the $E_{2}$ (high) mode becomes dominant, accompanying a very weak intensity of $A_{1}$ (TO) mode at $378 \mathrm{~cm}^{-1}$ and a negligible signal of the peak at $331 \mathrm{~cm}^{-1}$. The relative intensity of the individual peaks in the $E_{\perp}$ configuration is similar to the Raman shift signatures of the $\mathrm{ZnO}$ nanorod array, which were grown along the $c$ axis. In contrast, for the $\bar{X}(Z, Z) X$ configuration, where the incident/scattered light has the polarization parallel to the $c$ axis of the nanorod (denoted as $E_{\|}$), the peak at $378 \mathrm{~cm}^{-1}$ corresponding to the $A_{1}$ (TO) mode becomes dominant with a large suppression of the $E_{2}$ (high) mode at $438 \mathrm{~cm}^{-1}$. In addition, the peak of $A_{1}$ (acoustic overtone) at $331 \mathrm{~cm}^{-1}$ also persists well in the $E_{\|}$polarization condition. The Raman intensity $I$ can be usually expressed as $I \sim\left|\hat{e}_{s} \cdot \stackrel{\leftrightarrow}{R} \cdot \hat{e}_{l}\right|^{2}$, where $\stackrel{\leftrightarrow}{R}$ is the Raman tensor and $e_{l}=\cos \varphi \hat{X}$ $+\sin \varphi \sin \theta \hat{Y}+\sin \varphi \cos \theta \hat{Z}, \quad e_{s}=\cos \varphi \hat{X}+\sin \varphi \sin \beta \hat{Y}+\sin$ $\varphi \cos \beta \hat{Z}, \hat{e}_{l}\left(\hat{e}_{s}\right)$ is the unit vector of the incident (scattered) light polarization and $\theta$ (or $\beta$ ) is the angle between the incident (or scattered) light polarization and the nanorod axis. In our case, $\phi=\pi / 2$ corresponds to the angle between the propagating direction of scattered light and the orientation of the nanorod axis. According to the theoretical prediction, ${ }^{11,12}$ when $\theta=\beta$, the intensity of the $A_{1}$ (TO) at $378 \mathrm{~cm}^{-1}$ should become dominant when $\theta=0^{\circ}$ and the $E_{2}$ (high) mode at $438 \mathrm{~cm}^{-1}$ should be dominant when $\theta=90^{\circ}$, which shows a good consistency with the above observation in the $\bar{X}(Z, Z) X$ and $\bar{X}(Y, Y) X$ configurations, respectively. However, the nonzero signal at $438 \mathrm{~cm}^{-1}$ (or $378 \mathrm{~cm}^{-1}$ ) in the $\bar{X}(Y, Y) X$ (or $\bar{X}(Z, Z) X)$ configuration is mainly attributed to nonperfect 

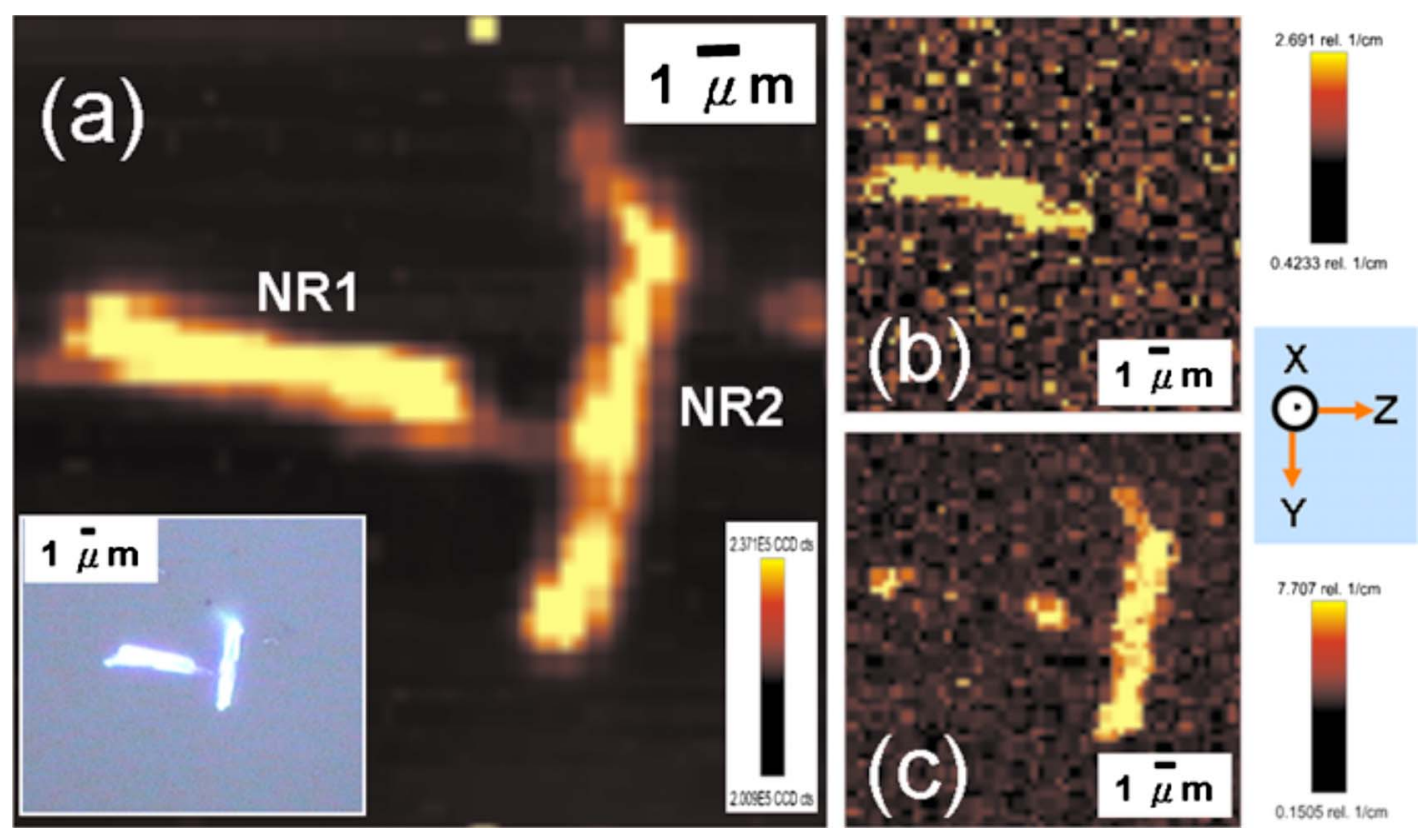

FIG. 3. (Color online) (a) Raman scattering mapping image of two individual $\mathrm{ZnO}$ nanorods with orientation almost perpendicular to each other. The inset in (a) shows the optical image of $\mathrm{ZnO}$ nanorods. (b) Raman images generated by integration of $\left(370-385 \mathrm{~cm}^{-1}\right)$ and (c) $\left(430-445 \mathrm{~cm}^{-1}\right)$ spectral ranges for the $X(Z, Z) \bar{X}$ polarization configuration.

the incident/scattered light. As the polarization configuration is changed to $\bar{X}(Z, Y) X$, all the $A_{1}$ (TO), $E_{1}$ (TO), and $E_{2}$ (high) modes appear. The above result clearly accounts for the nature of polarization-dependent anisotropy in the Raman spectra of an individual $\mathrm{ZnO}$ nanorod.

We have further chosen two individual $\mathrm{ZnO}$ nanorods with orientations almost perpendicular to each other to perform the Raman scattering image mapping experiment. The inset in Fig. 3(a) shows the optical microscopic image of the two nanorods. By intensity integration of the spectral domain ranging from 250 to $900 \mathrm{~cm}^{-1}$, the Raman image of the two individual $\mathrm{ZnO}$ nanorods, labeled as NR 1 (nanorod 1) and NR 2 (nanorod 2), can be obtained, as shown in Fig. 3(a). By integrating the intensity of the spectral domain $\left(370-385 \mathrm{~cm}^{-1}\right)$ around the $A_{1}$ (TO) mode at $378 \mathrm{~cm}^{-1}$ and of the spectral domain $\left(430-445 \mathrm{~cm}^{-1}\right)$ around the $E_{2}$ (high) mode at around $438 \mathrm{~cm}^{-1}$ in the $\bar{X}(Z, Z) X$ configuration, the complementary images with the maximum signals almost on the portion of the NR1 and NR2 are obtained, as shown in Figs. 3(b) and 3(c), respectively. The polarization of incident light is now pointing along the scale bar, as shown in Fig. 3(a), which is assigned as the $Z$ axis. The result provides the information correlating the local phonon behavior with geometric and orientation-dependent anisotropy. However, the nonvanishing Raman intensity image at the two ends of the NR1 in Fig. 3(c) is possibly due to (i) the nonperfect orientation of the NR1 or nonuniform crystal growth along the $c$ axis with respect to the incident photon polarization or (ii) the boundary mismatch of the dielectric function $\varepsilon$ between a nanorod and its surroundings (air), which may also cause the strong dependence of the transmission of the light inside a nanorod on its shape, orientation and size..$^{14,15,20}$

In summary, an individual $\mathrm{ZnO}$ nanorod has demonstrated a large anisotropy in its Raman scattering spectra when different incident/scattered light polarization configurations are used. The Raman image mapping signals provide direct information accounting for the anisotropic phonon behavior of the individual $\mathrm{ZnO}$ nanorods.

This work is supported by the National Science Council, Taiwan (Project Nos. 96-2112-M-002-030-MY3 and NSC 96-2120-M-001-001). The authors would also like to thank Dr. Li-Chyong Chen for providing the SEM images.

${ }^{1}$ H. J. Ko, Y. F. Chen, Z. Zhu, T. Yao, I. Kaobayyashi, and H. Uchiki, Appl. Phys. Lett. 76, 1905 (2000).

${ }^{2}$ W. I. Park, Y. H. Jun, S. W. Jung, and G.-C. Yi, Appl. Phys. Lett. 82, 964 (2003).

${ }^{3}$ D. M. Bagnall, Y. F. Chen, Z. Zhu, T. Yao, S. Koyama, M. Y. Shen, and T. Goto, Appl. Phys. Lett. 70, 2230 (1997).

${ }^{4}$ P. Zu, Z. K. Tang, G. K. L. Wong, M. Kawasaki, A. Ohtomo, H. Koinuma, and Y. Segawa, Solid State Commun. 103, 459 (1997).

${ }^{5}$ H. Co, J. Y. Xu, E. W. Seelig, and R. P. H. Chang, Appl. Phys. Lett. 76, 2997 (2000).

${ }^{6}$ D. Snoke, Science 273, 1351 (1996).

${ }^{7}$ J. D. Joannopoulos, P. R. Villeneuve, and S. Fan, Nature (London) 386, 143 (1997).

${ }^{8}$ C. W. Chen, K. H. Chen, C. H. Shen, A. Ganguly, L. C. Chen, J. J. Wu, H. I. Wen, and W. F. Pong, Appl. Phys. Lett. 88, 241905 (2006).

${ }^{9}$ J. C. Johnson, H. Yan, P. Yang, and R. J. Saykally, J. Phys. Chem. B 107, 8816 (2003).

${ }^{10}$ P. J. Pauzauskie, D. Talaga, K. Seo, P. Yang, and F. Lagugne-Labarthet, J. Am. Chem. Soc. 127, 17146 (2005).

${ }^{11}$ T. Livneh, J. Zhang, G. Cheng, and M. Moskovits, Phys. Rev. B 74, 035320 (2006).

${ }^{12}$ C. L. Hsiao, L. W. Tu, T. W. Chi, M. Chen, T. F. Young, C. T. Chia, Y. M. Chang, Appl. Phys. Lett. 90, 043102 (2007).

${ }^{13}$ A. Hartschuh, E. Sanchez, X. S. Xie, and L. Novotny, Phys. Rev. Lett. 90, 095503 (2003).

${ }^{14}$ J. Frechette and C. Carraro, J. Am. Chem. Soc. 128, 14774 (2006).

${ }^{15}$ H. M. Fan, X. F. Fan, Z. H. Ni, Z. X. Shen, Y. P. Feng, and B. S. Zou, J. Phys. Chem. C 112, 1865 (2008).

${ }^{16}$ H. Harima, J. Phys.: Condens. Matter 14, R967 (2002).

${ }^{17}$ T. C. Damen, S. P. S. Porto, and B. Tell, Phys. Rev. 142, 570 (1966).

${ }^{18}$ M. Rajalakshmi, A. K. Arora, B. S. Bendre, and S. Mahamuni, J. Appl. Phys. 87, 2445 (2000).

${ }^{19}$ R. P. Wang, G. Xu, and P. Jin, Phys. Rev. B 69, 113303 (2004).

${ }^{20}$ L. Cao, B. Nabet, and J. E. Spanier, Phys. Rev. Lett. 96, 157402 (2006). 